\title{
Are Children and Adolescents More Susceptible to the New Strains of SARS-Cov-2
}

\section{Than the Earlier Ones?}

\author{
Mohammad Abu Bashar* \\ Department of Community Medicine, Post Graduate Institute of Medical Education and Research, India
}

*Corresponding author: Mohammad Abu Bashar, Department of Community Medicine, Post Graduate Institute of Medical Education and Research, Chandigarh, India

\section{Abstract}

The clinical impact of the new SARS-CoV-2 lineage B.1-1.7 on children and young people (aged 18 years or younger) regarding acute respiratory COVID-19 is yet to be fully defined. Media reports of increases in admissions to hospital and more serious illness in children and young people have resulted in public confusion and implicated the B.1.1.7 variant as a more pathogenic infection within this group.

\section{Introduction}

According to the Centres for Disease Control and Prevention (CDC), it has been reported that children are less susceptible to develop severe manifestations of COVID-19; however, they can be infected with the virus, get sick from COVID-19, and spread the virus [1]. The majority of the children infected with COVID-19 have been reported to have mild or no symptoms. However, with the emergence of new variants of SARS-CoV-2, concerns have been raised regarding the susceptibility of the infection from these new variants among the paediatric population.

\section{Current evidence}

According to the $C D C$, there are currently five variants of concerns (VOCs) in the United States, including B.1.1.7 (UK variant), B.1.351 (South African variant), P.1 (initially detected in Brazil), and B.1.427 and B.1.429 (both of which were first identified in California, US) [2]. Studies have also shown that these variants, including 501Y.V1 (B.1.1.7) in UK, P.1 (501Y.V3) in Brazil, and 501Y.V2 (B.1.351) in South Africa, contribute to increased transmission of COVID-19. According to the report of Davies et al.3, the UK variant has been estimated to be more transmissible than the precursor lineage but has no clear evidence for a change in disease severity; however, the greater transmission will lead to higher incidence and more hospital admissions [3].

The clinical impact of the new SARS-CoV-2 lineage
B.1.1.7 on children and young adults (under the age of 18 years) with acute respiratory COVID-19 has yet to be determined. Also, it has been hypothesized in the earlier reports that the paediatric population might be more susceptible to infections with VOC as compared to the original strain of SARS-CoV-2; however, till date, no such evidence has come up. Media reports of increases in hospital admissions and more serious illnesses in children and young people have caused public chaos and panic, and the latest variants, especially the B.1.1.7 variant, have been implicated in causing a more pathogenic infection in this population. However, recent data show that B.1.1.7 has not changed the paediatric hospitalization rate and that severe COVID-19 remains rare among the kids. No significant differences were found with respect to age, the proportion of patients with comorbidities, the proportion of patients from Black, Asian, and minority ethnic background, or deprivation score between groups denoting first and second waves [4]. There was also no evidence of more severe disease in children and adolescents during the second wave of COVID-19, implying that the infection with the B.1.1.7 variant might have a similar clinical course to infection with the original strain.

\section{Conclusion}

No evidence of more severe disease has occurred in children and young people during the second wave, suggesting that infection with the B.1.1.7 variant does not result in an appreciably different clinical course to the 
original strain. Severe acute respiratory COVID-19 remains an uncommon occurrence in children and young people.

\section{References}

1. Centers for Disease Control and Prevention (CDC). COVID-19 in Children and Teens.

2. Centers for Disease Control and Prevention (CDC). About Variants of the
Virus that Causes COVID-19.

3. Davies NG, Abbott S, Bernard R (2021) Estimated transmissibility and impact of SARS-CoV-2 lineage B.1.1.7 in England. Science 372(6538): eabg3055.

4. Brookman S, Cook J, Zucherman M (2021) Effect of the new SARS-CoV-2 variant B.1.1.7 on children and young people. Lancet Child Adolesc Health 5(4): e9-e10. 